\title{
Erratum to: Observation of Crossover from Ballistic to Diffusion Regime for Excimer Molecules in Superfluid ${ }^{4} \mathbf{H e}$
}

\author{
D.E. Zmeev • F. Pakpour • P.M. Walmsley • \\ A.I. Golov · P.V.E. McClintock · S.N. Fisher • \\ W. Guo - D.N. McKinsey - G.G. Ihas · W.F. Vinen \\ Published online: 10 October 2012 \\ (c) Springer Science+Business Media New York 2012
}

\section{Erratum to: J Low Temp Phys \\ DOI 10.1007/s10909-012-0720-6}

F. Pakpour's name is correct as presented in this erratum.

The online version of the original article can be found under doi:10.1007/s10909-012-0720-6.

D.E. Zmeev (凶) · F. Pakpour · P.M. Walmsley · A.I. Golov

School of Physics and Astronomy, University of Manchester, Oxford Road, Manchester M13 9PL, UK

e-mail: dzmeev@gmail.com

D.E. Zmeev P. P.E. McClintock · S.N. Fisher

Department of Physics, Lancaster University, Lancaster LA1 4YB, UK

W. Guo · D.N. McKinsey

Department of Physics, Yale University, P.O. Box 208120, New Haven, CT 06520-8120, USA

G.G. Ihas

Department of Physics, University of Florida, P.O. Box 118440, Gainesville, FL 32611-8440, USA

W.F. Vinen

School of Physics and Astronomy, University of Birmingham, Birmingham B15 2TT, UK 